\title{
Diffusion Tensor Imaging on Teenagers, Born at Term With Moderate Hypoxic-ischemic Encephalopathy
}

\author{
ZOLTAN NAGY, KATARINA LINDSTROM, HELENA WESTERBERG, STEFAN SKARE, \\ JESPER ANDERSSON, BOUBOU HALLBERG, ANDERS LILJA, OLOF FLODMARK, \\ HUGO LAGERCRANTZ, TORKEL KLINGBERG, AND ELISABETH FERNELL \\ Department of Woman and Child Health, Neonatal Unit [Z.N., H.L.], Neuropediatric Unit [Z.N., H.W., \\ T.K., E.F.], Astrid Lindgren Children's Hospital Q2:07; Department of Neuropediatrics [K.L.], \\ Department of Neonatology [B.H], Department of Clinical Neuroscience [J.A., S.S.], Department of \\ Neuroradiology [A.L., O.F.], Karolinska University Hospital, 171 76, Stockholm, Sweden
}

\begin{abstract}
Hypoxic-ischemic encephalopathy (HIE) is graded with three levels of severity-mild, moderate and severe. The outcome of individuals with mild and severe grades can be reliably predicted from this scheme. Individuals with moderate degree are divided in outcome between those who suffer major neurologic problems (e.g., cerebral palsy) and those who are assumed to recover from the incident. It is however not clear if the recovery is complete and unquestionable.

A group of adolescents who had been born at term, diagnosed with moderate HIE but had not developed cerebral palsy, were investigated with diffusion tensor imaging. Fractional anisotropy maps were used as a basis of comparison to a group of controls of the same age and gender distribution.

In several white matter areas fractional anisotrophy was lower in the group of individuals with a history of moderate HIE. These areas include the internal capsules (bilaterally in the posterior
\end{abstract}

limb and on the right in the anterior limb), the posterior and anterior corpus callosum as well as frontal inferior white matter areas.

These results indicate that even in the absence of such major neurologic impairments as cerebral palsy, moderate HIE causes long term white matter disturbances which are not repaired by adolescence. (Pediatr Res 58: 936-940, 2005)
CP, cerebral palsy
Abbreviations
DTI, diffusion tensor imaging
FA, fractional anisotropy
HIE, hypoxic-ischemic encephalopathy
MRI, magnetic resonance imaging
PVL, periventricular leukomalacia

Hypoxic-ischemic encephalopathy (HIE) in term-born infants is diagnosed with three grades (I-III) of severity - mild, moderate and severe respectively $(1,2)$. This grading system reliably predicts outcome for grade I (good prognosis) and grade III (severe neurohandicap or death) $(3,4)$. However, for children with a grade II (moderate) HIE the outcome is divided. A certain proportion will develop cerebral palsy (CP), while the rest are considered to have a good outcome. There are indications pointing to physical and mental impairment by school age (5). although a more recent study found no such unfavourable outcome $(6,7)$.

Received July 8, 2004; accepted January 24, 2005.

Correspondence: Zoltan Nagy, Karolinska Institute, Department of Woman and Child Health, Neonatal Unit, Astrid Lindgren Children's Hospital Q2:07, 17176 Stockholm, Sweden, Phone: +46-8-517-77354, Fax: +46-8-517-77353; e-mail: zoltan.nagy@ kbh.ki.se.

Supported by grants from Stiftelsen Frimurare Barnhuset i Stockholm, Sällskapet Barnavård, Swedish Research Council (VR), Stiftelsen Samariten and the Swedish Society of Medicine.

DOI: 10.1203/01.pdr.0000186516.85702.61
Following a systematic review of follow-up studies, Dilenge et al. concluded (a) that $30-50 \%$ of children with moderate HIE had mental deficits at follow-up and (b) that giving a prognosis in the neonatal period is difficult if it is based on outcome studies which are done at less than $5 \mathrm{y}$ of age (8).

In a previous Swedish study of over forty-thousand live infants, the children were followed up until 18 mo of age and about half of those with moderate HIE developed CP while the remainder were without such impairments (9). The presented work is focused on the outcome of surviving, term-born children with at least moderate HIE.

There is a large body of work using conventional magnetic resonance imaging (MRI) to investigate the immediate and short-term effects of perinatal HIE. These studies indicate the involvement of cortical and subcortical grey matter as well as specific white matter regions (10). For example Rutherford et al. reported extensive white matter abnormalities in children with perinatal HIE whom had abnormal outcome at $2 \mathrm{y}$ of age (11). Furthermore, abnormal signal intensity on MRI examinations within the posterior limb of the internal capsule is a good 
predictor of poor outcome (12). Also, in an animal model perinatal asphyxia was shown to result in the reduction of the corpus callosum (13).

Based on these previous results we hypothesized that the subgroup of teenagers with moderate HIE but without CP would have disturbances in white matter regions when compared with controls without HIE. The method of investigation was diffusion tensor imaging (DTI), a specialized MRI technique that is specific for the scrutiny of the white matter (14).

\section{METHODS}

Subjects. The children were selected through a nation-wide search in Sweden. The inclusion criteria were a birth year of 1985, full-term birth, at least a moderate degree of HIE. and the absence of clinical signs of CP. The HIE classification was made retrospectively and independently by two of the authors (K.L and B.H).

There were a total of 97468 live births in Sweden in 1985. Of these children we identified 684 who were full-term born with an Apgar score $<7$ at $5 \mathrm{~min}$. On a retrospective evaluation of the records, 45 of these 684 children could be classified (2). to have HIE with at least moderate severity. Nineteen children had developed CP and in six children such data were unknown. This study focuses on the remaining 20 children. Of these one had dwarfism and was not included. Thus 19 families were contacted with inquiries to participate in the study. Eleven families agreed. The data from two children were later excluded (one with a cyst and one with an unsuccessful DTI). Thus nine children were included in the statistical analyses. Eight of these had moderate HI. The records of one participant were difficult to classify, indicating a borderline between moderate and severe HIE.

All these nine adolescents had seizures in the neonatal period. A neonatal cranial ultrasound had been performed in 8 of the 9 children. One had a slight dilatation of the ventricles and a minor cyst, interpreted to be posthaemorrhagic. Two had initial signs of oedema with thinning of the lateral ventricles but the subsequent ultrasound examinations were normal. The remaining 5 participants had normal results.

The participants underwent an IQ test (WISC) at the time of the MRI examination. Three of these 9 adolescents had a score below 70, a further three were between 70 and 85 while the remainder over 85 . Still 8 of the 9 participants had cognitive problems, mainly with memory and attention.

The control subjects were selected from the Stockholm region with inclusion criteria of a birth weight $\geq 2500 \mathrm{~g}$ and a gestational age $\geq 37 \mathrm{wk}$ and having no perinatal complications or obvious neurologic problems. Furthermore, they were matched to the study group for age and gender. There were 4 boys in each group and the ages at the time of MRI scans were $17.15 \mathrm{y}$ (SD
$0.40 \mathrm{y}$ ) and $17.44 \mathrm{y}$ (SD $0.48 \mathrm{y}$ ) for the group of individuals with moderate HIE and control group respectively.

The study was approved by the Ethical Committee of the Karolinska Hospital in Stockholm, Sweden. The participants were introduced to the procedure and possible dangers of an MRI examination after which they gave informed consent.

MRI data acquisition. All subjects underwent a scanning session on a 1.5-Tesla General Electric Signa Echospeed (Milwaukee, WI, USA) scanner, employing a single shot, diffusion-weighted, echo planar imaging sequence which was triggered with pulse gating. The inplane resolution was $1.72 \mathrm{~mm} \times$ $1.72 \mathrm{~mm}$. From each subject 36 slices were collected, each with a $3 \mathrm{~mm}$ thickness. The diffusion scheme included 20 images with noncollinear diffusion gradients $\left(b=1000 \mathrm{~s} / \mathrm{mm}^{2}\right)$ and 4 nondiffusion-weighted images $(b=0$ $\mathrm{s} / \mathrm{mm}^{2}$ ).

Eight of the nine teenagers with HIE also underwent a series of clinical scans which were performed in their respective local hospitals within Sweden. In the one remaining case there were technical difficulties and the examination could not be performed. Two of the eight individuals were scanned with a 3T scanner while the others with 1.5 scanners. For each individual these clincal scans included sagittal T1-weighted images and axial T2-weighted images. A coronal FLAIR (fluid attenuated inversion recovery) sequence was done for all but one of the individuals. Furthermore, according to local protocols and availabilities coronal T2-weighted images, axial T1-weighted images and coronal FIR (fast inversion recovery) images were also collected for a subset of individuals. All the images were evaluated by a qualified neuroradiologist (A.L.) at Karolinska Institute. The details are presented in table 1.

Fractional anisotropy images. In DTI, the imaging sequence is sensitized to water molecular motion. From the data obtained one can calculate fractional anisotropy (15) (FA), a measure with microstructural, histologic correlates as described in Fig. 1.

To calculate FA, first eddy current distortions and head movement were corrected (16). Subsequently, the diffusion tensor was estimated from which the FA was calculated for each voxel (15).

Subject movement can lead to artifacts. As an indicator of subject movement the mean of the absolute value of the rotations along the $\mathrm{x}, \mathrm{y}$ and $\mathrm{z}$ axes was used. These values could be obtained from the movement correction algorithm. Using rotations to quantify movement is reasonable as translations are usually difficult in the supine position with the head immobilized. Separate $t$ tests were used for each of the movement variables but no group differences were found at a confidence level of $p<0.05$.

Spatial normalization (17) was performed with the SPM software (http:// www.fil.ion.ucl.ac.uk/spm/). The mean of the five nondiffusion-weighted images for each subject was normalized to a common template and then we used these normalization parameters to register the FA-images to a common space for all subjects. These normalized FA images form the basis of comparison between the study group and the controls. The analysis was based on a $t$ test, employed a correction for multiple comparisons and was carried out

Table 1. Results of clinical evaluation.

\begin{tabular}{|c|c|c|c|c|c|c|c|}
\hline Subject & $\begin{array}{c}\text { Sagittal } \\
\text { T1 }\end{array}$ & Axial T2 & Coronal T2 & Coronal FLAIR & Coronal FIR & Axial T1 & Other \\
\hline 1 & - & $\begin{array}{r}\text { Small periventricular } \\
\text { high signal lesions }\end{array}$ & Not done & $\begin{array}{l}\text { Small periventricular lesions \& } \\
\text { reduction of periventricular } \\
\text { white matter }\end{array}$ & Not done & - & Not done \\
\hline 2 & - & - & - & & - & Not Done & Not done \\
\hline 3 & - & - & Not done & & Not done & - & Not done \\
\hline 4 & - & - & Not done & - & - & Not Done & Not done \\
\hline 5 & - & $\begin{array}{l}\text { Small periventri-cular } \\
\text { high signal lesions at } \\
\text { trigones bilaterally }\end{array}$ & $\begin{array}{l}\text { Similar lesions to } \\
\text { Axial } \mathrm{T} 22 \mathrm{~mm} \text { in } \\
\text { diameter. }\end{array}$ & Not done & Not done & - & $\begin{array}{l}\text { Axial FLAIR Small } \\
\text { periventricular } \\
\text { lesions similar to } \\
\text { Axial T2 }\end{array}$ \\
\hline 6 & - & - & - & - & - & Not Done & GRE T2*- \\
\hline 7 & - & $\begin{array}{l}\text { Small periventricular } \\
\text { white matter lesions } \\
\text { and wide } \\
\text { peri-vascular spaces }\end{array}$ & Not done & $\begin{array}{l}\text { Slight reduction of } \\
\text { periventricular white matter } \\
\text { \& small white matter lucence }\end{array}$ & Not done & $\begin{array}{l}\text { Wide perivascular } \\
\text { spaces at left } \\
\text { trigone }\end{array}$ & Not done \\
\hline 8 & - & $\begin{array}{l}\text { Occipital lobe cortical } \\
\text { and subcortical small } \\
\text { gliotic/atrophic } \\
\text { lesions }\end{array}$ & Not done & $\begin{array}{l}\text { Occipital lobe subcorctical } \\
\text { gliosis mainly left }\end{array}$ & Not done & $\begin{array}{l}\text { Occipital cortical } \\
\text { widening }\end{array}$ & $\begin{array}{l}\text { Coronal T1 Occipital } \\
\text { cortical widening }\end{array}$ \\
\hline
\end{tabular}

A no comment (-) means a normal finding. Note: here GRE T2 ${ }^{*}$ means gradient echo T2-star. 


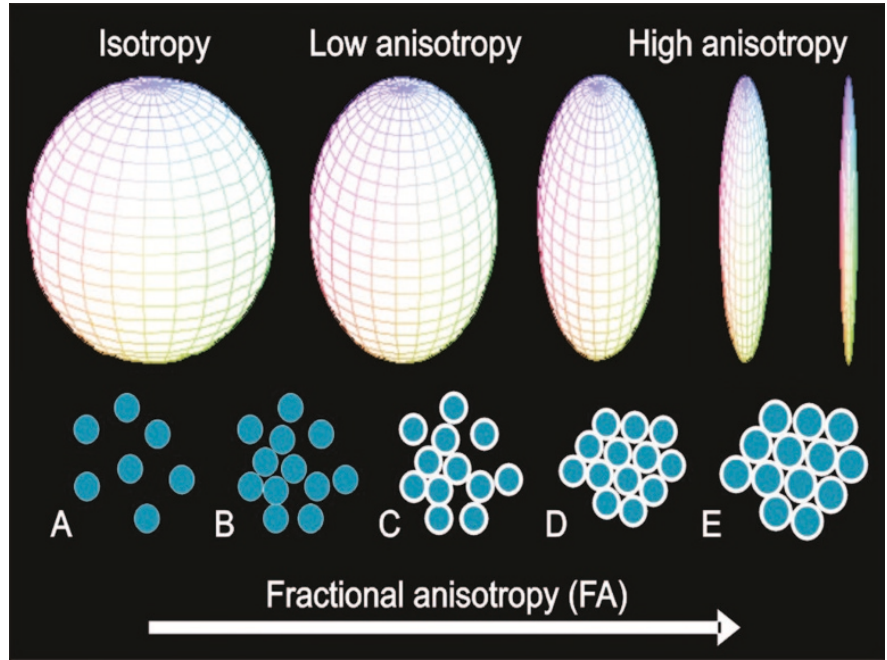

Figure 1. Description of fractional anisotropy. Diffusion tensor imaging measures extracellular water diffusion. When water can freely diffuse it does so in a spherically symmetric manner which is termed isotropic diffusion (top left). Anisotropy is a term given to diffusion which is not spherically symmetric (anisotropy increases from left to right at the top row), the extent of which can be measured by fractional anisotropy. In the second row the circles illustrate the cross section of axons. When the axons are far apart (A) diffusion tensor imaging indicates isotropic diffusion. That is due to the short times involved in MRI imaging during which the water molecules do not travel far enough to collide with axons and hence can diffuse equally well in all directions. As the axons become more numerous (B), acquire myelin (C), become more tightly packed (D) and increase in diameter (E) the diffusion of extracellular water becomes highly anisotropic (diffusion would be easier perpendicular to the page - i.e., along the axons). These histologic changes would be measured by an increase in fractional anisotropy (indicated by the thick arrow at the bottom). Evidently FA is zero for isotropic diffusion (i.e., the fraction of anisotropic diffusion is zero) and approaches the value of one as the diffusion becomes more and more ellipsoid in shape.

using the SPM software $(18,19)$. The comparison was confined to only those voxels with FA $>0.15$ because this threshold has been found to reliably isolate white matter from the rest of the brain. (20). Once the white matter was isolated, a voxel-wise search identified a subset of voxels where the individuals with moderate HIE had a lower FA.

It could be possible that the spatial normalization causes false positive results. To exclude this possibility, a separate analysis was made in which we also searched for voxels where the individuals with moderate HIE would have a higher FA (i.e., against our hypothesis). This search however did not result in statistically significant differences between the two groups, hence indicating that the results are not consequences of an erroneous anatomical normalization.

\section{RESULTS}

DTI results. The teenagers with moderate HIE had significantly lower FA values in several distinct brain regions. These areas included (a) the posterior limbs of the internal capsule bilaterally, (b) the anterior limb of the right internal capsule, (c) the posterior corpus callosum involving both the isthmus and the splenium (d) the right side of the genu as well as (e) inferior, frontal white matter regions bilaterally (Fig. 2). This fractional anisotropy reduction indicates that, in these specific regions, adolescents without major neurologic symptoms, such as $\mathrm{CP}$, but having a history of moderate HIE can have smaller, fewer, less well organized and less well myelinated axons (or combination of any of these factors).

In general our findings could have been confounded by shortcomings in the normalization process. To validate our

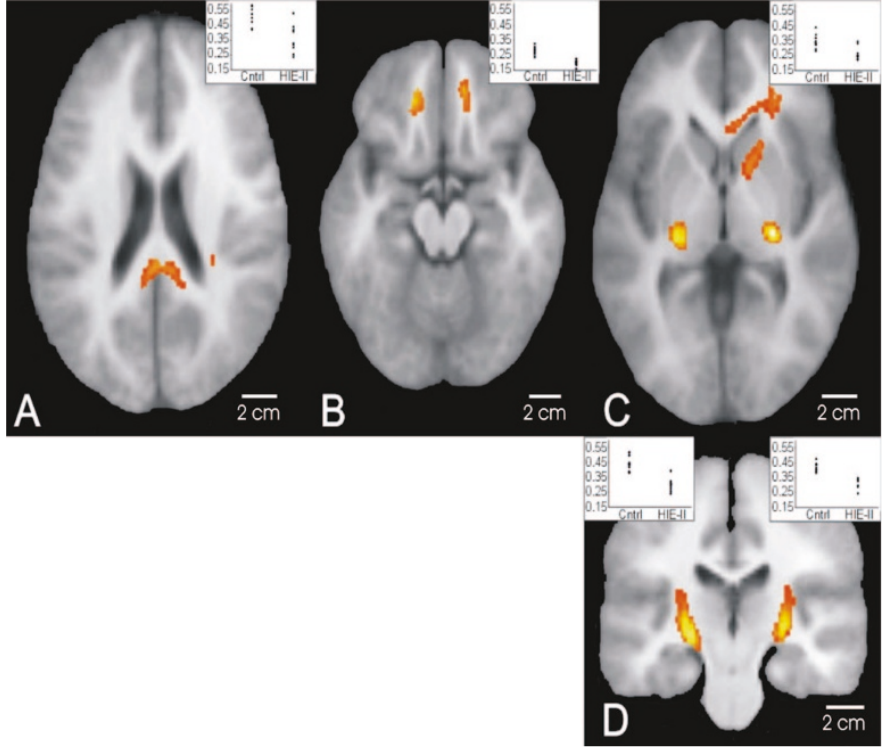

Figure 2. Fractional anisotropy group differences. Regions where fractional anisotropy is lower for the individuals with moderate HIE are displayed (A-D). All the regions are statistically significant below 0.003 but the significance increases as the colour becomes more yellow. The results are displayed on the mean normalized 3-D SPGR image of all the subjects. For the inset scatter plots FA is along the $y$ axis. (A) Posterior corpus callosum. (B) Inferior frontal white matter. The inset only indicates the FA values of the significant cluster of voxels on the right. The cluster on the left had similar FA values. (C) Right anterior corpus callosum (genu), right anterior limb of the internal capsule and the posterior limbs of the internal capsule. The inset indicates FA values in the anterior internal capsule. The FA values for the anterior corpus callosum are not given specifically. They are slightly lower than that of the posterior corpus callosum in (A). (D) Coronal view through the posterior internal capsules. The scatter plots indicate the FA values in the internal capsules of the corresponding side.

methods we statistically compared the nondiffusion weighted (T2) images on a voxel-by-voxel basis (recall that white matter appears dark on a T2-weighted image). These were the same images we used for the spatial normalization. We did not find any voxels where $\mathrm{T} 2$ signal intensity was higher in the controls. On the other hand we located several areas where T2 signal intensity was significantly lower (i.e., more specific to white matter) for that group. As expected, these areas were located at the edges between ventricle and corpus callosum and around the major fibre pathways. These are the areas where partial volume problems usually arise. However, the voxels where T2-signal intensity was lower (i.e., more specific to white matter) for the controls were entirely different voxels from those in which FA values were higher (i.e., better white matter microstructure) for the controls. Therefore, although there were slight, systematic differences between the groups (i.e., where the controls had lower T2 signal intensity), these differences are not likely to have affected our findings in a way to create false positives.

Clinical imaging findings. Two of the eight teenagers with clinical images could be suspected of having periventricular leukomalacia (PVL), which indicates asphyxia before the $35^{\text {th }}$ week of gestation (subjects \#1 and \#5, table 1). One additional teenager was classified as borderline PVL (subject \#7 table 1). Another teenager had cortical and subcortical changes indica- 
tive of partial perinatal asphyxia but showed no signs of PVL (subject \#8, table 1) The detailed findings are summarized in table 1 .

\section{DISCUSSION}

Our major finding was that children with moderate HIE but without CP have lower FA values in several white matter regions, including the posterior limbs of the internal capsule, anterior and posterior corpus callosum and anterior white matter regions inferiorly.

Characteristic changes can be seen in the posterior limbs of the internal capsule on clinical MRI examination with a histologic correlate of oedema (10). In oedematous white matter the axons are more separated and hence the diffusion can increase in the direction perpendicular to them (Fig. 1). This explains well the reduction in FA soon after the injury. However, oedema usually recedes after the primary injury or at least normal signal intensity on $\mathrm{T} 1$ and $\mathrm{T} 2$ images returns. Therefore, oedema at adolescence probably explains to a lesser extent the lower FA we see in the individuals with moderate HIE at adolescence. This however does not exclude the possibility of initial oedema causing un-reparable damage to the axons and myelin. The abnormal MRI signal in the posterior limb of the internal capsules, observed shortly after birth, in children with moderate HIE predicts poor neurodevelopment at $1 \mathrm{y}$ of age. The sensitivity and specificity in that study were found to be 0.90 and 1.0 respectively (12). The authors however considered all children with moderate HIE (i.e., not only a subgroup without cerebral palsy as in the present study) and followed them up only to $1 \mathrm{y}$ of age. Therefore, it is possible that children who seemed normal at $1 \mathrm{y}$ of age could develop neurologic problems at a later stage in life. Dilenge et al. specifically state the difficulties in neonatal prognosis based on follow-up studies which are done at less than $5 \mathrm{y}$ of age (8). Hence our findings imply that the initial injury to the internal capsule may not resolve itself fully (at least not until adolescence) even if the injury is minor (i.e., not evident on clinical MRI) and even if it does not result in early neurologic impairment. Furthermore it is also plausible that perinatal injury elsewhere in the brain could result in the reduction of FA values in this area.

Corpus callosum injury has previously been found in association with moderate HIE. In one recent study a clear decrease of cross sectional area was demonstrated in the genu (most anterior region) and the isthmus (second most posterior region) of the corpus callosum (21). Visual inspection suggests that these regions correspond to those with decreased FA as presented here. Regional reduction of corpus callosum size has also been observed in children with focal lesions (22). Therefore region-specific alterations in the corpus callosum do occur and, according to our findings, FA can be used to reliably identify the specific locations of injury.

There is evidence that the corpus callosum develops until the middle of the $3^{\text {rd }}$ decade in life (23-25). However, age related changes cannot be responsible for our findings as the groups were well matched for age. Also, there has been a debate about the dimorphism of corpus callosum size and development. Our results should be safe from these effects (even if true) because the groups in our study were perfectly matched for gender.

In addition to conventional MRI, which localizes white matter, DTI also indicates the direction of white matter fibre tracks. Knowledge of the trajectories of white matter tracks may help to explain the decline in cognitive, motor and sensory abilities in relation to the sites of injuries. It is well known that within the corpus callosum axons run between the hemispheres and that the posterior limb of the internal capsule form the cortico-spinal tracks. It is less well known that the anterior limb of the internal capsule consists of white matter tracks which run in the anteroposterior direction (26). However, fibre directions in all these regions can be confirmed by DTI (Fig. $3 \mathrm{~A})$. In addition FA images are also displayed, which indicate that in the above mentioned regions diffusion is highly anisotropic due to the high density of parallel and mostly myelinated fibres (Fig. 3B). FA values are lower in the anterior white matter but the predominant directionality of axons is anteroposterior. The reduction in FA is possibly due to the branching of fibres which no longer run in parallel fashion but still target the frontal regions (hence displayed in red, which indicates anteroposterior fibre directions).

It is also interesting that while moderate HIE affects the most posterior region of the internal capsule, preterm birth causes a reduction in FA in the anterior third of the posterior limb of the internal capsule (27). The axons in these separate regions probably originate in different cortical regions, and hence must subserve different functional areas. The ability to identify these grey matter areas would be invaluable but at present it is not

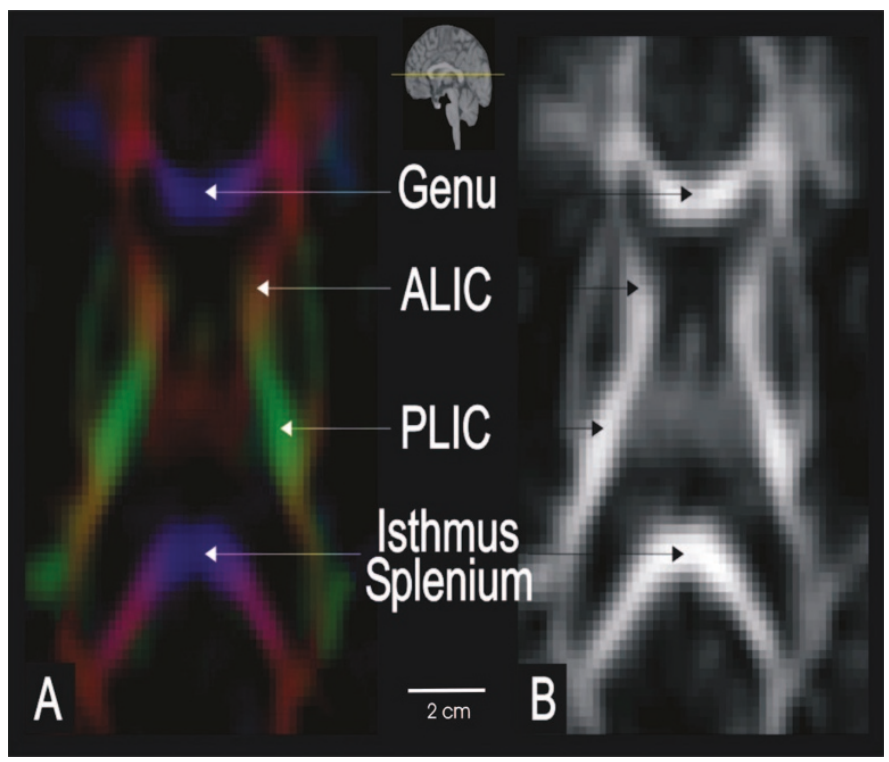

Figure 3. Color coded white matter directions and FA images. The inset indicates the level of horizontal sections displayed in A and B. Genu $=$ most anterior region of the corpus callosum, ALIC $=$ anterior limb of the internal capsule, PLIC $=$ posterior limb of the internal capsule, Splenium $=$ the most posterior portion of the corpus callosum, Isthmus = region of the corpus callosum just anterior of the splenium. (A) The colors indicate the direction of white matter fibres with blue $=$ left-right, red $=$ anteroposterior and green $=$ cranial-caudal (point out from the plane of the page). (B) The intensity corresponds to FA. Black corresponds to FA $=0$ and the higher the FA value the whiter the image. 
possible. Future research, combining DTI with fMRI and possibly other neuro-imaging modalities, may be able to answer these questions.

Prevention of HIE after hypoxic-ischemic insult is not yet possible. Although there is extensive research aimed in this direction, many of the currently researched methods are either difficult to achieve in clinical settings or are with questionable utility and possibly worse side effects. One method which shows promise is the possibility of cooling either the head or the entire body (28).

There are limitations to this study. First, out of the 26 available children we could only image 11 and obtained useful images in only 9 . The authors believe that these 9 children may be considered good representatives of the entire group because they came randomly from all parts of the country, were of the same age, were included with a very specific etiology and there were mainly practical reasons for those families who declined participation (such as unable to travel to Stockholm). Although this claim may be challenged, the results presented here should promote similar studies, in larger and different populations because it is unlikely that these results would be restricted to only those individuals imaged here. Secondly, we only found unilateral FA differences between the two groups in the anterior limb of the internal capsule and the genu of the corpus callosum. These may be due to the small number of subjects in each group. However, we included all the children that were available to us within the entire region of Sweden. Therefore improvements in this respect are not possible.

We conclude that even in the absence of such major neurologic problems as $\mathrm{CP}$, moderate HIE causes long-term disturbances in specific white matter regions. The lower FA in these white matter regions may indicate that this group of children have fewer, smaller, less well myelinated or less well organized axons. As white matter tracts serve to connect specific cortical and sub-cortical grey matter regions these disturbances may be responsible for impaired cognitive abilities of these children which have been reported previously. We recommend that normal outcome is not declared for this subgroup in the neonatal period but longer follow-up is given, including appropriate measures when needed.

Acknowledgments. The authors thank Christina Malmqvist for generously sparing her time to improve the quality of MRI scans and the comfort of the subjects in this study.

\section{REFERENCES}

1. Sarnat HB, Sarnat MS 1976 Neonatal encephalopathy following fetal distress. A clinical and electroencephalographic study. Arch Neurol. 33:696-705
2. Levene MI, Sands C, Grindulis H, Moore JR 1986 Comparison of two methods of predicting outcome in perinatal asphyxia. Lancet. 1:67-69

3. Robertson C, Finer N 1985 Term infants with hypoxic-ischemic encephalopathy: outcome at 3.5 years. Dev Med Child Neurol. 27:473-484

4. Fenichel GM 1983 Hypoxic-ischemic encephalopathy in the newborn. Arch Neurol. 40:261-266

5. Robertson CM, Finer NN, Grace MG 1989 School performance of survivors of neonatal encephalopathy associated with birth asphyxia at term. J Pediatr. 114:753760

6. Kjellmer I, Beijer E, Carlsson G, Hrbek A, Viggedal G 2002 Follow-up into young adulthood after cardiopulmonary resuscitation in term and near-term newborn infants I. Educational achievements and social adjustment. Acta Paediatr. 91:1212-1217

7. Viggedal G, Lundalv E, Carlsson G, Kjellmer I 2002 Follow-up into young adulthood after cardiopulmonary resuscitation in term and near-term newborn infants. II. Neuropsychological consequences. Acta Paediatr. 91:1218-1226

8. Dilenge ME, Majnemer A, Shevell MI 2001 Long-term developmental outcome of asphyxiated term neonates. J Child Neurol. 16:781-792

9. Thornberg E, Thiringer K, Odeback A, Milsom I 1995 Birth asphyxia: incidence, clinical course and outcome in a Swedish population. Acta Paediatr. 84:927-932

10. Rutherford MA 2002 MRI of the neonatal brain. W.B. Saunders, London, pp 201-224.

11. Rutherford M, Pennock J, Schwieso J, Cowan F, Dubowitz L 1996 Hypoxicischaemic encephalopathy: early and late magnetic resonance imaging findings in relation to outcome. Arch Dis Child Fetal Neonatal Ed. 75:F145-F151

12. Rutherford MA, Pennock JM, Counsell SJ, Mercuri E, Cowan FM, Dubowitz LM, Edwards AD 1998 Abnormal magnetic resonance signal in the internal capsule predicts poor neurodevelopmental outcome in infants with hypoxic-ischemic encephalopathy. Pediatrics. 102:323-328

13. Kohlhauser C, Mosgoller W, Hoger H, Lubec B 2000 Myelination deficits in brain of rats following perinatal asphyxia. Life Sci. 67:2355-2368

14. Le Bihan D 2003 Looking into the functional architecture of the brain with diffusion MRI. Nat Rev Neurosci. 4:469-480

15. Basser PJ, Pierpaoli C 1996 Microstructural and physiological features of tissues elucidated by quantitative-diffusion-tensor MRI. J Magn Reson B. 111:209-219

16. Andersson JL, Skare S 2002 A model-based method for retrospective correction of geometric distortions in diffusion-weighted EPI. Neuroimage. 16:177-199

17. Ashburner J, Friston KJ 1999 Nonlinear spatial normalization using basis functions. Hum Brain Mapp. 7:254-266

18. Friston KJ, Holmes AP, Worsley KJ, Poline JP, Frith CD, Frackowiak RS 1994 Statistical parametric maps in functional imaging: a general linear approach. Hum Brain Mapp. 2:189-210

19. Friston KJ, Worsley KJ, Frackowiak RS, Mazziotta JC, Evans AC 1993 Assessing the significance of focal activations using their spatial extent. Hum Brain Mapp. 1:210-220

20. Jones DK, Simmons A, Williams SC, Horsfield MA 1999 Non-invasive assessment of axonal fiber connectivity in the human brain via diffusion tensor MRI. Magn Reson Med. 42:37-41

21. Maneru C, Junque C, Salgado-Pineda P, Serra-Grabulosa JM, Bartres-Faz D, RamirezRuiz B, Bargallo N, Tallada M, Botet F 2003 Corpus callosum atrophy in adolescents with antecedents of moderate perinatal asphyxia. Brain Inj. 17:1003-1009

22. Moses P, Courchesne E, Stiles J, Trauner D, Egaas B, Edwards E 2000 Regional size reduction in the human corpus callosum following pre- and perinatal brain injury. Cereb Cortex. 10:1200-1210

23. Pujol J, Vendrell P, Junque C, Marti-Vilalta JL, Capdevila A 1993 When does human brain development end? Evidence of corpus callosum growth up to adulthood. Ann Neurol. 34:71-75

24. Keshavan MS, Diwadkar VA, DeBellis M, Dick E, Kotwal R, Rosenberg DR, Sweeney JA, Minshew N, Pettegrew JW 2002 Development of the corpus callosum in childhood, adolescence and early adulthood. Life Sci. 70:1909-1922

25. Giedd JN, Rumsey JM, Castellanos FX, Rajapakse JC, Kaysen D, Vaituzis AC, Vauss YC, Hamburger SD, Rapoport JL 1996 A quantitative MRI study of the corpus callosum in children and adolescents. Brain Res Dev Brain Res. 91:274-280

26. Felderhoff-Mueser U, Rutherford MA, Squier WV, Cox P, Maalouf EF, Counsell SJ, Bydder GM, Edwards AD 1999 Relationship between MR imaging and histopathologic findings of the brain in extremely sick preterm infants. AJNR Am J Neuroradiol. 20:1349-1357

27. Nagy Z, Westerberg H, Skare S, Andersson JL, Lilja A, Flodmark O, Fernell E, Holmberg K, Bohm B, Forssberg H, Lagercrantz H, Klingberg T 2003 Preterm children have disturbances of white matter at 11 years of age as shown by diffusion tensor imaging. Pediatr Res. 54:672-679

28. Whitelaw A, Thoresen M 2002 Clinical assessment and therapeutic interventions for hypoxic-ischemic encephalopathy in the full-term infant. In: Lagercrantz $\mathrm{H}$, Hanson M, Evrard P, Rodeck C (eds) The Newborn Brain: neuroscience and clinical applications. Cambridge University Press, Cambridge, pp 415-442. 